\title{
Six-headed coracobrachialis muscle
}

\author{
N. Zielinska ${ }^{1}$, Ł. Olewnik ${ }^{1}$ (1) \\ Department of Anatomical Dissection and Donation, Medical University of Lodz, Poland
}

[Received: 29 May 2021; Accepted: 19 July 2021; Early publication date: 24 August 2021]

\begin{abstract}
The coracobrachialis muscle is the smallest muscle of the anterior compartment of the arm. It is responsible for flexion and abduction in the glenohumeral joint. The coracobrachialis muscle is morphologically variable both in its insertion and origin. Moreover, some additional heads or structures may also occur. The present report describes a six-headed coracobrachialis muscle originated as a common junction with the short head of the biceps brachii muscle from the coracoid process. All of these heads insert into the medial surface of the humeral shaft. It is important to note that the musculocutaneous nerve was piercing the fourth belly. Other heads were innervated by branches from the musculocutaneous nerve. Knowledge of the morphological variability of this muscle is essential not only for anatomists but for clinicians as well. (Folia Morphol 2022; 81, 3: 809-813)
\end{abstract}

Key words: coracobrachialis muscle, morphological variations, compression, additional head, quadriceps femoris, biceps brachii, embryology, clinical implications

\section{INTRODUCTION}

Coracobrachialis muscle (CBM) belongs to the anterior group of the arm region. Its origin is located on the coracoid process but is represented by a common junction with the short head of the biceps brachii muscle (SHBB). Its distal attachment is located on the medial surface and border of the body of the humerus. Three parts of the CBM can be distinguished: the proximal, middle and distal part. The last one is the largest and the most superficial $[6,25]$. The CBM is innervated by the musculocutaneous nerve (MCN), piercing this muscle in most cases [23]. Oxygenated blood is delivered to the CBM by muscular branches of the brachial artery [25].

The CBM is responsible for flexion and abduction in the glenohumeral joint. Additionally, it is involved in providing stabilisation of the humeral head [15]. However, apart from its anatomical function, progress in science allows surgeons to use the CBM during reconstruction of the breast (after mastectomy) or axillary malformations. The CBM may be also used in the treatment of long-standing facial palsy $[10,12]$.

There are numerous morphological variations of the CBM. For example, in some cases, an additional head occurs. Olewnik et al. [20] found a four-headed CBM. Another case was described by Catli et al. [7], in which the CBM was represented by three heads. Szewczyk et al. [23] created a classification system basing on the number of heads and its site of proximal attachment. In the available literature, we can also find descriptions of accessory structures connected with the CBM, like the coracobrachialis longus muscle (CBL) [18], coracocapsularis muscle [25], coracobrachialis brevis muscle [18], or the minor coracobrachial muscle of Cruveilhier [9].

The occurrence of an additional structure usually is connected with some kind of compression of neural or vascular elements [11]. One of the examples is the

Address for correspondence: Ł. Olewnik, DPT, PhD, Ass. Prof., Department of Anatomical Dissection and Donation, Medical University of Lodz, ul. Żeligowskiego 7/9, 90-136 Łódź, Poland, e-mail: lukasz.olewnik@umed.lodz.pl

This article is available in open access under Creative Common Attribution-Non-Commercial-No Derivatives 4.0 International (CC BY-NC-ND 4.0) license, allowing to download articles and share them with others as long as they credit the authors and the publisher, but without permission to change them in any way or use them commercially. 
compression of the MCN, when it courses between two CBM heads $[18,23]$. It is responsible for motor innervation of muscles from the anterior compartment of the arm. It may lead to weakness of these muscles and some problems in flexion and abduction in the glenohumeral joint. On the other hand, the MCN provides sensory innervation to the elbow joint, lateral part of the forearm, and lateral part of the hand. In the case of $\mathrm{MCN}$ compression, tingling or numbness may occur in these regions [18].

The present report describes a six headed CBM originated as a common junction with the SHBB from the coracoid process. All heads insert into the medial surface of the humeral shaft. It is important to note that the MCN was piercing the fourth head. Knowledge of the morphological variability of this muscle is essential for all clinicians. To our knowledge, this is the first description of such a case.

\section{Ethical approval and consent to participate}

The cadaver belonged to the Department of Anatomical Dissection and Donation, Medical University of Lodz, Poland.

\section{CASE REPORT}

An 81-year-old body donor (height $183 \mathrm{~cm}$ ) was subjected to routine anatomical dissection for research and teaching purposes at the Department of Anatomical Dissection and Donation. The donor did not have any surgeries in the upper limb area. The right upper limb was subjected to traditional anatomical dissection [17, 26-28], and a morphological variation of a number of CBM heads was found (Fig. 1).

In the present case, the CBM was represented by six distinct heads which created a proximal attachment with the SHBB as a common junction. Such an origin was located on the coracoid process. In this place, the common junction was $12.25 \mathrm{~mm}$ wide and $2.61 \mathrm{~mm}$ thick.

All distinct heads had a distal attachment on the medial surface of the humeral shaft. The first head was arising from the common junction, and its length was $32.27 \mathrm{~mm}$. In its proximal part, it was $8.08 \mathrm{~mm}$ wide and 2.08 thick. The width of its insertion was $6.85 \mathrm{~mm}$ and thickness was $2.55 \mathrm{~mm}$.

The second head was $5.03 \mathrm{~mm}$ wide and $2.00 \mathrm{~mm}$ thick in the place of division. Its distal attachment was $5.38 \mathrm{~mm}$ wide and $2.60 \mathrm{~mm}$ thick. The length of this head was $32.29 \mathrm{~mm}$.

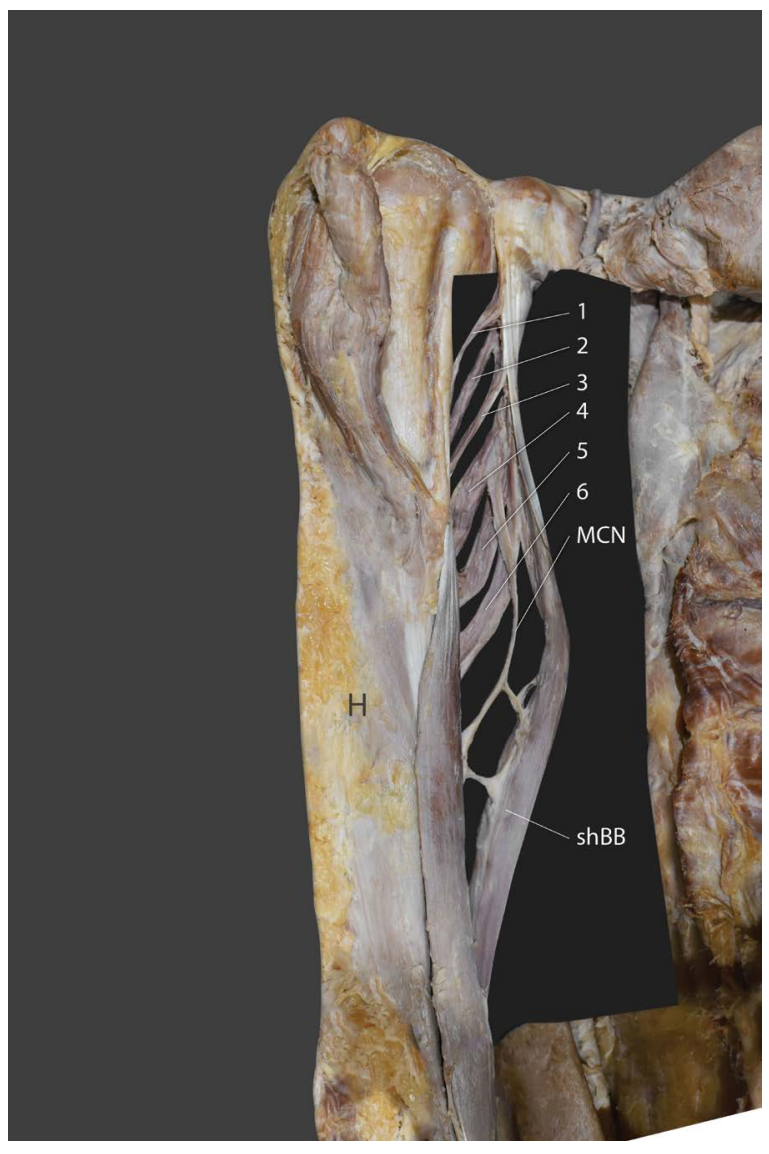

Figure 1. Six-headed coracobrachialis muscle. Right upper limb; $\mathrm{H}$ - humerus; shBB — short head of the biceps brachii; MCN musculocutaneous nerve; 1 - the first head of the coracobrachialis muscle; 2 - the second head of the coracobrachialis muscle; 3 - the third head of the coracobrachialis muscle; 4 - the fourth head of the coracobrachialis muscle; 5 - the fifth head of the coracobrachialis muscle; 6 - the sixth head of the coracobrachialis muscle.

The width of the third head in its proximal part was $5.30 \mathrm{~mm}$. The thickness of this head in the same place was $2.60 \mathrm{~mm}$. The length from this place to the insertion was really similar to the previous one $-33.19 \mathrm{~mm}$. The distal attachment was the widest of all and equalled $15.34 \mathrm{~mm}$. The thickness in this place was $3.67 \mathrm{~mm}$.

The fourth head was $32.84 \mathrm{~mm}$ long. In the point of the division, the width was $10.14 \mathrm{~mm}$ and the thickness was $4.88 \mathrm{~mm}$. Next, its distal attachment was $10.04 \mathrm{~mm}$ wide and $5.67 \mathrm{~mm}$ thick (it was the thickest insertion).

The length of the fifth head was $36.18 \mathrm{~mm}$. It was $6.57 \mathrm{~mm}$ wide and $1.67 \mathrm{~mm}$ thick in its proximal part arising from the common junction with the SHBB. In its attachment to the bone, it was $7.88 \mathrm{~mm}$ wide and $3.62 \mathrm{~mm}$ thick. 
Table 1. Morphometric measurements of individual heads of the coracobrachialis muscle

\begin{tabular}{|c|c|c|c|c|c|c|}
\hline & $1^{\text {st }}$ head & $2^{\text {nd }}$ head & $3^{\text {rd }}$ head & $4^{\text {th }}$ head & $5^{\text {th }}$ head & $6^{\text {th }}$ head \\
\hline Length & $32.27 \mathrm{~mm}$ & $32.29 \mathrm{~mm}$ & $33.19 \mathrm{~mm}$ & $32.84 \mathrm{~mm}$ & $36.18 \mathrm{~mm}$ & $47.92 \mathrm{~mm}$ \\
\hline Origin & \multicolumn{6}{|c|}{ Common junction with the SHBB } \\
\hline Width & \multicolumn{6}{|c|}{$12.25 \mathrm{~mm}$} \\
\hline Thickness & \multicolumn{6}{|c|}{$2.61 \mathrm{~mm}$} \\
\hline \multicolumn{7}{|l|}{ Division* } \\
\hline Width & $8.08 \mathrm{~mm}$ & $5.03 \mathrm{~mm}$ & $5.30 \mathrm{~mm}$ & $10.14 \mathrm{~mm}$ & $6.57 \mathrm{~mm}$ & $7.13 \mathrm{~mm}$ \\
\hline Thickness & $2.08 \mathrm{~mm}$ & $2.00 \mathrm{~mm}$ & $2.60 \mathrm{~mm}$ & $4.88 \mathrm{~mm}$ & $1.67 \mathrm{~mm}$ & $2.22 \mathrm{~mm}$ \\
\hline Insertion & \multicolumn{6}{|c|}{ Medial surface of the shaft of the humerus } \\
\hline Width & $6.85 \mathrm{~mm}$ & $5.38 \mathrm{~mm}$ & $15.34 \mathrm{~mm}$ & $10.04 \mathrm{~mm}$ & $7.88 \mathrm{~mm}$ & $11.36 \mathrm{~mm}$ \\
\hline Thickness & $2.55 \mathrm{~mm}$ & $2.60 \mathrm{~mm}$ & $3.67 \mathrm{~mm}$ & $5.67 \mathrm{~mm}$ & $3.62 \mathrm{~mm}$ & $3.93 \mathrm{~mm}$ \\
\hline
\end{tabular}

*Division of the common junction for distinct heads of the coracobrachialis muscle; SHBB — short head of the biceps brachii muscle

The last head appeared to be the longest and equalled $47.92 \mathrm{~mm}$. Its width in the proximal part was $7.13 \mathrm{~mm}$, while the thickness in the same place was $2.22 \mathrm{~mm}$. The insertion of this head was $11.36 \mathrm{~mm}$ wide and $3.93 \mathrm{~mm}$ thick.

It is important to point out that the MCN was piercing the fourth head. In this place, a diameter of the MCN nerve was $6.66 \mathrm{~mm}$. Some small branches from the MCN innervated distinct heads of the CBM.

An electronic calliper (Mitutoyo Corporation, Kawasaki-shi, Kanagawa, Japan) was used to complete all necessary measurements. Each measurement was repeated twice with an accuracy of up to $0.1 \mathrm{~mm}$.

No other morphological variabilities were found. Table 1 shows the morphometric measurements of the presented case.

\section{DISCUSSION}

The presence of one additional head has been described in literature many times. Moreover, there are some muscles which may be represented by few accessory bellies, and such case reports usually describe very rare structures. For example, the fifth head of the quadriceps femoris (QF) is nothing special. Willan et al. [24], in 1990, carried out a study in which one additional head of the QF was present in about one third of the limbs. On the other hand, Ruzik et al. [22] found the QF with seven heads. Two additional muscle bellies were composed of tendons, and another one was a tensor of the vastus intermedius [22]. Olewnik et al. [19] created the first classification system of additional heads of the QF, in which the most common (75\%) was five-headed QF. Moreover, they found six-headed (21\%), seven-headed (2.9\%), and eight-headed (1.9\%) QF [19].
Another muscle with high morphological variability is the biceps brachii (BB). In the literature, we can find some descriptions of one additional head. For example, Mujahid Ansari et al. [16] found a case of three-headed BB. However, Ballesteros et al. [1] carried out a study in which the results showed that one additional head of the $\mathrm{BB}$ is not a rare variant because it occurred in $21 \%$ of specimens. Moreover, there are some cases of four-headed BB. Catli et al. [7] detected the BB with two supernumerary heads, originated in various places. A very interesting case was a five-headed BB, described by Je et al. [13]. One supernumerary head originated from the anterior surface of the distal tendinous part of the pectoralis major muscle. The other two originated from the body of the humerus [13].

Coming back to the CBM, the situation is similar. There are some descriptions of one extra head of the CBM, like this found by Gabrelotti et al. [11]. To confirm the statement that one additional head is usually nothing special, we should look at the results of the study carried out by Szewczyk et al. [23]. It turned out that the CBM was represented by a double muscle belly in $42.6 \%$ of specimen. Catli et al. [7] described a rare variant of the CBM, which was characterized by two additional heads. In the study carried out by Szewczyk et al. [23], we can also find a description of such a structure, and its prevalence was around $8 \%$ (8/101 upper limbs). Two of these supernumerary heads originated from the coracoid process and the last one originated from the SHBB [23]. There is also a description of four-headed CBM, and such a structure has not been found by Szewczyk et al. [23]. The CBM with additional three heads was described 
by Olewnik et al. [21]. All heads were innervated by the MCN [21].

Our present case is something like the eight-headed QF or five-headed BB. It originated as a common junction with the SHBB from the coracoid process. All six heads had their distal attachment on the medial surface of the humeral shaft. The reason for the occurrence of such a complex structure is astonishing and most likely is associated with changes occurring during the embryological growth. In the first stage, brachalis muscle, BB, and CBM muscles are tightly connected in a common pre-muscle mass. When embryos achieve the length of 14 to $16 \mathrm{~mm}$, these three muscles can be recognized as distinct structures [2-4]. Probably, additional heads of the CBM are formed at this stage of embryonic development (14-16 mm) or maybe this embryological process lasts longer and the single mass of the CBM divides into smaller parts recognized as distinct supernumerary heads.

Looking for embryological development of QF and its additional heads, our hypothesis seems to make sense. QF is represented by a single mass overlying the anterolateral region when embryo is $11 \mathrm{~mm}$ long. Then the differentiation occurs and in the 20-mm embryo four distinct structures of the QF may be distinguished [2-4]. Similarly, additional heads may be created from 11 to $19 \mathrm{~mm}$ of embryonal length. However, there is also a possibility that this process occurs later, because the muscle mass is smaller than that observed in standard parts of the QF. However, our statement is only a hypothesis, so a further embryological research should be performed to confirm it.

The presence of an additional head is usually connected with some clinical implications. A supernumerary head of the CBM may compress the MCN, especially when this nerve courses between two bellies [18, 23]. The MCN is responsible for motor innervation of muscles from the anterior compartment of the arm, so its entrapment may cause some problems with flexion and abduction in the glenohumeral joint. Structures like the elbow joint, lateral parts of the forearm and hand receive sensory innervation from the MCN, so the compression of the MCN may manifest in tingling or numbness [18]. However, in our case, the MCN was piercing the fourth head of the CBM, and in our opinion, the possibility of entrapment is lower.

The occurrence of an extra head of the CBM may be important not only for anatomists but also for clinicians. Upper limb injuries are very common, which is connected with various (sometimes really invasive) surgical operations. For that reason, some results of computed tomography or magnetic resonance showing such variations may confuse a surgeon. It is even worse if the change is detected during an operation. This may cause some complications or prolong the surgery [14]. Moreover, if additional heads occur only unilaterally, it can result in asymmetry between right and left side, and sometimes it may be wrongly recognized as some kind of tumour [8].

As mentioned above, not only variations connected with the number of heads take place. For example, Szewczyk et al. [23] classified the CBM, basing on its different place and representation of insertion, and distinguished two types. The first one was represented by a single insertion onto the distal $1 / 3$ of the humerus. In turn, the second one was characterized by a double insertion: one to the distal $1 / 3$ of the humerus (as the previous one), and one which created fusion with the medial head of the triceps brachii [23]. Moreover, they also divided the CBM basing on the course of the MCN. Type I was represented by the nerve piercing the muscle belly. Type II was represented by the MCN passing between heads of the CBM [23]. In the present case, only the fourth head was pierced by this nerve and some small branches innervated other heads.

There are also some interesting muscles connected with the CBM. For example, the CBM brevis, whose origin is located on the anterior surface of the coracoid process. It may insert onto the lesser tuberosity or surgical neck of the humerus [5]. A special kind of the CBM brevis is the coracocapsularis muscle, whose proximal attachment is fused with the capsule of the shoulder joint [25]. Another example of high variability of the CBM is coracobrachialis minor secundus which is characterized by a connection with the pectoralis major tendon [6], or coracobrachial muscle of Cruveilhier, also represented by fusion, but with the latissimus dorsi muscle [9]. A really rare structure is a CBM longus whose point of insertion may be variable [18].

Summing up this section, due to high morphological variability of the CBM, there is a possibility to find some interesting structures which have not been described in the literature yet.

\section{CONCLUSIONS}

The CBM is associated with high morphological variability. All of these variations seem to be impor- 
tant not only for anatomists but also for clinicians. The most possible reason for the occurrence of the six-headed coracobrachialis muscle presented in this case report is an improper embryological development; however, further embryological investigations should be performed to confirm this phenomenon.

\section{Conflict of interest: None declared}

\section{REFERENCES}

1. Ballesteros LE, Forero PL, Buitrago ER. Evaluation of additional head of biceps brachii: a study with autopsy material. Folia Morphol. 2014; 73(2): 193-198, doi: 10.5603/ FM.2014.0028, indexed in Pubmed: 24902098.

2. Bardeen C. Studies of the development of the human skeleton. Am J Anat. 1905: 265-302.

3. Bardeen C. Development and variation of the nerves and the musculature of the inferior extremity and of the neighboring regions of the trunk in man. Am J Anat. 2005; 6(1): 259-390, doi: 10.1002/aja.1000060108.

4. Bardeen C. Development and variation of the nerves and the musculature of the inferior extremity and of the neighboring regions of the trunk in man. Am J Anat. 2005; 6(1): 259-390, doi: 10.1002/aja.1000060108.

5. Bauones S, Moraux A. The accessory coracobrachialis muscle: ultrasound and MR features. Skeletal Radiol. 2015; 44(9): 1273-1278, doi: 10.1007/s00256-015-2153-1, indexed in Pubmed: 25924580.

6. Bergman R, Afifi A, Miyauchi R. Illustrated encyclopedia of human anatomic variations. Anatomy Atlas 2017.

7. Catli MM, Ozsoy U, Kaya Y, et al. Four-headed biceps brachii, three-headed coracobrachialis muscles associated with arterial and nervous anomalies in the upper limb. Anat Cell Biol. 2012; 45(2): 136-139, doi: 10.5115/ acb.2012.45.2.136, indexed in Pubmed: 22822469.

8. Cheema P, Singla R. Low incidence of the third head of the biceps brachii in the north indian population. J Clin Diagnostic Res. 2012; 5: 1323-1326.

9. Cruveilhier J. Traité d'anatomie descriptive I. Asselin, Paris 1862.

10. El-Naggar MM, Al-Saggaf S. Variant of the coracobrachialis muscle with a tunnel for the median nerve and brachial artery. Clin Anat. 2004; 17(2): 139-143, doi: 10.1002/ ca.10213, indexed in Pubmed: 14974102.

11. Garbelotti SA, Marques SR, Rocha PR, et al. An unusual case of accessory head of coracobrachialis muscle involving lateral cord of brachial plexus and its clinical significance. Folia Morphol. 2017; 76(4): 762-765, doi: 10.5603/FM.a2017.0033, indexed in Pubmed: 28353299.

12. Georgiev GP, Tubbs RS, Landzhov B. Coracobrachialis longus muscle: humeroepitrochlearis. Cureus. 2018; 10(5): e2615, doi: 10.7759/cureus.2615, indexed in Pubmed: 30027007.

13. Je SS, Park B, Kim J, et al. Five-headed biceps brachii muscle with a rare origin from the tendon of pectoralis major muscle. Anat Sci Int. 2016; 91(1): 110-113, doi: 10.1007/ s12565-015-0288-8, indexed in Pubmed: 26012790.

14. Kopuz C, Içten N, Yildirim M. A rare accessory coracobrachialis muscle: a review of the literature. Surg Radiol Anat. 2003; 24(6): 406-410, doi: 10.1007/s00276-002-0079-5, indexed in Pubmed: 12652369.
15. Moore KL, Agur A, Dalley A. Clinically Oriented Anatomy. Lippincott Williams\&Wilkins, 2013.

16. Mujahid Ansari M, Gupta UK, Laique Ahmed M, et al. Third head of biceps brachii with anatomical consideration and clinical implication: a case report. J Evol Med Dent Sci. 2013; 2(6): 630-634, doi: 10.14260/jemds/314.

17. Olewnik $\measuredangle$, Karauda $P$, Gonera $B$, et al. Impact of plantaris ligamentous tendon. Sci Rep. 2021; 11(1): 4550, doi: 10.1038/s41598-021-84186-w, indexed in Pubmed: 33633305.

18. Olewnik $Ł$, Paulsen F, Tubbs RS, et al. Potential compression of the musculocutaneous, median and ulnar nerves by a very rare variant of the coracobrachialis longus muscle. Folia Morphol. 2021; 80(3): 707-713, doi: 10.5603/ FM.a2020.0085, indexed in Pubmed: 32844391.

19. Olewnik $Ł$, Tubbs RS, Ruzik K, et al. Quadriceps or multiceps femoris?-Cadaveric study. Clin Anat. 2021; 34(1): 71-81, doi: 10.1002/ca.23646, indexed in Pubmed: 32644202.

20. Olewnik $\measuredangle$, Zielinska N, Karauda P, et al. The co-occurrence of a four-headed coracobrachialis muscle, split coracoid process and tunnel for the median and musculocutaneous nerves: the potential clinical relevance of a very rare variation. Surg Radiol Anat. 2021; 43(5): 661-669, doi: 10.1007/s00276-020-02580-x, indexed in Pubmed: 32979058.

21. Olewnik $\measuredangle$, Zielinska N, Karauda P, et al. The co-occurrence of a four-headed coracobrachialis muscle, split coracoid process and tunnel for the median and musculocutaneous nerves: the potential clinical relevance of a very rare variation. Surg Radiol Anat. 2021; 43(5): 661-669, doi: 10.1007/ s00276-020-02580-x, indexed in Pubmed: 32979058.

22. Ruzik K, Waśniewska $A$, Olewnik $\measuredangle$, et al. Unusual case report of seven-headed quadriceps femoris muscle. Surg Radiol Anat. 2020; 42(10): 1225-1229, doi: 10.1007/ s00276-020-02472-0, indexed in Pubmed: 32318799.

23. Szewczyk B, Polguj M, Paulsen F, et al. A proposal for a new classification of coracobrachialis muscle morphology. Surg Radiol Anat. 2021; 43(5): 679-688, doi: 10.1007/ s00276-021-02700-1, indexed in Pubmed: 33564931.

24. Willan PL, Mahon M, Golland JA. Morphological variations of the human vastus lateralis muscle. J Anat. 1990; 168: 235-239, indexed in Pubmed: 2323995.

25. Wood J. On human muscular variations and their relation to comperative anatomy. J Anat Physiol. 1867: 44-59.

26. Zielinska $N$, Olewnik $\measuredangle$, Karauda $P$, et al. A very rare case of an accessory subscapularis muscle and its potential clinical significance. Surg Radiol Anat. 2021; 43(1): 19-25, doi: 10.1007/s00276-020-02531-6, indexed in Pubmed: 32656573

27. Zielinska N, Szewczyk B, Tubbs RS, et al. Coexistence of two accessory flexor pollicis longus heads or coexistence of two-headed flexor pollicis longus with an unrecognized anatomical structure? Surg Radiol Anat. 2021; 43(5): 763-769, doi: 10.1007/s00276-021-02721-w, indexed in Pubmed: 33656594.

28. Zielinska N, Tubbs RS, Podgórski M, et al. The subscapularis tendon: A proposed classification system. Ann Anat. 2021; 233: 151615, doi: 10.1016/j.aanat.2020.151615, indexed in Pubmed: 33068734. 
